Información farmacológica

REACCIONES ADVERSAS Anemia aplásica asociada con el cloranfenicol oftálmico y el nifedipino; infertilidad masculina y esteroides anabólicos; propionato de fluticasona (Flovent ${ }^{\circledR}$ ) y riesgo de trastornos eosinofílicos; adormecimiento repentino por consumo de pramipexol; trastornos hepáticos graves asociados con el uso de trovafloxacino y alatrofloxacino; acidosis láctica debida al consumo de metformina; graves efectos adversos por el uso de etanercepto (Enbrel ${ }^{\circledR}$ ).

POLÍTICA SOBRE MEDICAMENTOS

USO RACIONAL

RETIROS DEL MERCADO

ENMIENDAS A LA ROTULACIÓN DECISIONES DIVERSAS

PUBLICACIONES
Últimos reglamentos de la FDA sobre el uso de productos protectores contra los rayos solares; medidas de la FDA para controlar la venta por Internet de medicamentos que requieren receta médica; anuncios de la FDA sobre el carácter ilegal de la venta de productos de tabaco a menores; normas y procedimientos para el registro de productos naturales de uso medicinal en el Ecuador.

Uso indebido de suplementos dietéticos orales en forma líquida; Coumadin ${ }^{\circledR}$ y Marevan ${ }^{\circledR}$ no son intercambiables; se debe descartar el embarazo antes de prescribir clomifeno.

Retiro voluntario del sulfato de gentamicina inyectable; suspensión voluntaria del astemizol (Hismanal ${ }^{\circledR}$ ); retiro de todos los lotes del colutorio DawnMist ${ }^{\circledR}$; retiro de la poligelina por su asociación con hipotensión; retiro de productos del mercado argentino en junio de 1999; falsificación de Distensi ${ }^{\circledR}$ y de Buscapina Compositum ${ }^{\circledR}$ en el Perú.

Nueva rotulación y cambios en las indicaciones sobre el uso de la troglitazona (Rezulin ${ }^{\circledR}$ ).

Aprobada la temozolomida para tratar el cáncer cerebral; uso aprobado de una proteína de fusión para el tratamiento de linfomas; la talidomida (Thalomid ${ }^{\circledR}$ ) declarada medicamento huérfano para el tratamiento de la enfermedad de Crohn; aprobación de la rosiglitazona para el tratamiento de la diabetes tipo Il; adición de tiomersal a las vacunas.

World Health Organization. WHO Expert Committee on Specifications for Pharmaceutical Preparations: Thirty-fifth report. Geneva: WHO; 1999. (Serie de informes técnicos 885).

\section{REACCIONES ADVERSAS}

\section{Anemia aplásica por cloranfenicol oftálmico y nifedipino}

En colaboración con hematólogos de 17 hospitales del Área Metropolitana de Barcelona, el Institut Català de Farmacologia coordina un sistema de vigilancia epidemiológica de discrasias hemáticas graves. Aplicando un método de casos y controles de base poblacional, recientemente ha efectuado dos estudios de interés: uno sobre el posible riesgo de anemia aplásica asociado con el uso de cloranfenicol oftálmico, y el otro sobre ese riesgo en conexión con el uso de nifedipino.

En el primer estudio, basado en datos recogidos de manera sistemática, se calculó un riesgo relativo de 3,8 (intervalo de confianza de 95\%: 0,8 a 17) de anemia aplásica. Los resultados indican que no se puede descartar una asociación entre el uso de cloranfenicol oftálmico y la anemia aplásica. De haberla, sin embargo, el riesgo es muy bajo, de menos de 1 por 1000000 tratamientos.

En el segundo estudio, sobre el riesgo de anemia aplásica asociado con el uso de nifedipino, se encontró un riesgo relativo estadísticamente significativo de 4,9 (IC95\%: 1,8 a 10,6). Solo un paciente había tomado otro fármaco asociado con un riesgo de anemia aplásica (alopurinol) y ninguno había estado expuesto a benceno, disolventes o plaguicidas. También se calculó una incidencia de anemia aplásica entre pacientes tratados con nifedipino de 1,17 casos por 100000 años-usuario. Los resultados sugieren que el riesgo de anemia aplásica asociado con el uso de nifedipino es de la misma magnitud que el asociado con el uso de cloranfenicol por vía oral (1,7/100 000 expuestos) o de fenilbutazona $(2,2 / 100000)$. 


\section{Infertilidad masculina ocasionada por esteroides anabólicos (Reino Unido)}

Dos publicaciones británicas recientes describen nueve casos de infertilidad masculina ocasionada por esteroides anabólicos. Estos casos fueron similares en varios aspectos. Por lo general, los pacientes habían consultado por infertilidad y algunos estaban a punto de recurrir a la fertilización in vitro. En el examen físico tenían atrofia testicular y los recuentos de espermatozoides revelaron azoospermia en la mayor parte de los casos y oligospermia en los demás. Los espermatozoides también mostraban anormalidades morfológicas y alteraciones de la motilidad y las concentraciones séricas de testosterona y hormona foliculoestimulante (FSH) solían estar bajas.

La investigación de la infertilidad en un deportista debe considerar el posible uso de esteroides anabólicos. Esto tiene especial importancia porque al suprimirse estos medicamentos el recuento de espermatozoides suele normalizarse en pocos meses. Si no se tiene en cuenta esta posibilidad, podrían perderse mucho tiempo y dinero en pruebas y tratamientos innecesarios.

\section{Proprionato de fluticasona (Flovent ${ }^{\circledR}$ ) y trastornos eosinofílicos (Estados Unidos de América)}

La compañía Glaxo Wellcome notifica a los profesionales de la salud la aparición de algunos casos contados de trastornos eosinofílicos sistémicos, en ocasiones con síntomas de vasculitis compatibles con el síndrome de ChurgStrauss, en pacientes que inhalan propionato de fluticasona $\left(\right.$ Flovent $\left.{ }^{\circledR}\right)$.

\section{Pramipexol y adormecimiento repentino (Europa)}

La Agencia Europea para la Evaluación de Productos Medicinales (EMEA) ha emitido una advertencia sobre el uso de pramipexol, medica- mento antiparkinsoniano. Según la última información proporcionada por los comerciantes autorizados para vender el producto, su administración se asocia con episodios de adormecimiento súbito, que pueden producirse en cualquier momento después de la inhalación y a cualquier dosis dentro de los límites recomendados. Pueden ocasionar la muerte del paciente e incluso de terceros, a veces sin ningún síntoma previo. El adormecimiento repentino es diferente de la somnolencia o la sedación, habiéndose observado en algunos pacientes un deseo urgente e incontrolable de dormir. Según los datos disponibles, en la mayoría de los casos el adormecimiento repentino no se volvió a presentar después de reducir la dosis de pramipexol o de suspender el medicamento.

Tras su revisión inicial de estos datos, la EMEA quiere llamar la atención sobre estos episodios raros, pero impredecibles y potencialmente mortales.

- A los pacientes en tratamiento con pramipexol se les debe hacer una fuerte advertencia de que no manejen vehículos ni participen en otras actividades (tales como el manejo de maquinaria industrial) que conlleven un riesgo de traumatismo o muerte cuando no se está del todo alerta.

- Cualquier paciente que haya tenido adormecimiento repentino debe acudir a su médico de inmediato.

- Debido a la posibilidad de efectos aditivos, los pacientes que toman otros sedantes o que consumen alcohol junto con el pramipexol deben tomar precauciones.

El pramipexol fue autorizado en la Unión Europea en octubre de 1997 y en los Estados Unidos de América en julio de 1997.

\section{Hepatotoxicidad asociada con el uso de trovafloxacino y alatrofloxacino (Suiza y Estados Unidos de América)}

La Agencia Europea para la Evaluación de Productos Medicinales
(EMEA) ha publicado un comunicado de prensa con nueva información sobre dos nuevas fluoroquinolonas que acaban de ser autorizadas: trovafloxacino (Trovan ${ }^{\circledR}$, Turvel $^{\circledR}$ : Pfizer, Roerig) y alatrofloxacino (Trovan $\mathrm{IV}^{\circledR}$, Turvel IV ${ }^{\circledR}$ : Pfizer, Roerig). Desde febrero de 1998 se han documentado 140 casos de trastornos hepáticos graves, entre ellos ocho casos de defunción o trasplante hepático (cuatro de estos últimos con necrosis hepática). Según la revisión de los casos, en 35\% el trastorno hepático se acompañó de una reacción de hipersensibilidad. Las lesiones hepáticas aparecieron de 1 a 60 días después de iniciarse el tratamiento, lo cual indica que la aparición y gravedad de estas lesiones no se pueden predecir. A la luz de esta nueva información, el Comité para Medicamentos de Marca (Committee for Proprietary Medicinal Products, CPMP) ha empezado a reevaluar los riesgos de estos productos contra sus beneficios.

El trovafloxacino y el alatrofloxacino están indicados en los siguientes casos:

- El alatrofloxacino (concentrado de 5 $\mathrm{mg} / \mathrm{mL}$ para uso en solución para infusión, que viene en viales de 20, 40 y $60 \mathrm{~mL}$ ) actualmente se autoriza para el tratamiento endovenoso de la neumonía adquirida en la comunidad y de origen nosocomial (leve, moderada y grave); las infecciones intraabdominales con complicaciones y las infecciones pelvianas agudas; y las infecciones cutáneas y de los tejidos blandos con complicaciones.

- El trovafloxacino (comprimidos con cubierta entérica de 100 y $200 \mathrm{mg}$ ) se autoriza actualmente para el tratamiento por vía oral de la neumonía adquirida en la comunidad y la neumonía nosocomial (leve, moderada y grave); las exacerbaciones agudas de la bronquitis crónica; la sinusitis aguda; las infecciones intraabdominales complicadas y las infecciones pelvianas agudas; la salpingitis; la uretritis y la cervicitis gonocócicas sin complicaciones; la cervicitis por clamidias; y las infecciones cutáneas y de los tejidos blandos con complicaciones. 
La Administración de Alimentos y Medicamentos (FDA) de los Estados Unidos de América también ha emitido una advertencia sobre los riesgos de toxicidad hepática en conexión con el consumo de Trovan ${ }^{\circledR}$ (trovafloxacino oral) y Trovan IV ${ }^{\circledR}$ (alatrofloxacino para uso endovenoso). Esta medida fue ocasionada por informes de casos de hepatotoxicidad grave, algunos seguidos de muerte o trasplante hepático.

$\mathrm{Al}$ emitir esta advertencia, la FDA declara que Trovan ${ }^{\circledR}$ debe reservarse exclusivamente para los casos que cumplan todos los criterios siguientes:

- Pacientes con una o más infecciones específicas, tales como neumonía nosocomial o infecciones intraabdominales complicadas que, a criterio del médico, sean graves y pongan en peligro la vida o la integridad de un miembro.

- Pacientes que inician su tratamiento durante su internamiento en una institución (un hospital u hogar para ancianos).

- Pacientes cuyo médico opina que, aun teniendo en cuenta la información más reciente, los beneficios del producto pesan más que los riesgos que conlleva.

La FDA también advierte a los médicos que, en general, el tratamiento con Trovan $^{\circledR}$ no debe durar más de 14 días. Debe suspenderse incluso antes si el paciente tiene signos clínicos de disfunción hepática, como cansancio, pérdida del apetito, ictericia de piel y escleróticas, dolor abdominal intenso con náusea y vómitos u orina de color oscuro.

La FDA recomienda iniciar el tratamiento con Trovan ${ }^{\circledR}$ por la vía endovenosa en pacientes que cumplen todos los requisitos establecidos. Una vez que llegan a un estado clínico estable, pueden empezar a recibir el medicamento por la vía oral. Aunque es posible que en ciertos casos la vía de administración oral sea la adecuada desde que se inicia el tratamiento, la agencia insiste en que la forma oral del medicamento no debe usarse para tratar infecciones distintas de las indicadas.

No se han notificado casos de insuficiencia hepática, trasplante hepático o muerte como consecuencia de los trastornos hepáticos observados en los 7000 pacientes que fueron estudiados en los ensayos previos a la comercialización de $\operatorname{Trovan}^{\circledR}$. En julio de 1998, tras haber recibido informes de elevación de las enzimas hepáticas y de hepatitis sintomática en pacientes que habían recibido un tratamiento corto o prolongado, la FDA ayudó al fabricante a modificar la rotulación del producto a fin de subrayar el riesgo de trastorno hepático. Desde entonces, la FDA ha seguido recibiendo informes de toxicidad hepática, entre los que figuran algunos casos de mayor gravedad.

Actualmente la FDA sabe de 14 casos de insuficiencia hepática aguda que, según ha concluido, se asocian muy estrechamente con el medicamento. Seis de los pacientes fallecieron: cinco de insuficiencia hepática y otro después de un trasplante hepático (procedimiento que se practicó en cuatro pacientes en total). Tres sanaron sin necesidad de trasplante y en los dos casos adicionales el resultado final aún se desconoce.

\section{Acidosis láctica asociada con el uso de metformina (Francia)}

La acidosis láctica es una complicación poco frecuente del tratamiento con metformina, único medicamento contra la diabetes a base de biguanida que se vende en Francia. Casi la mitad de los pacientes que tienen esta complicación fallecen. Los estudios de farmacovigilancia efectuados en Europa muestran que la mayor parte de los casos de acidosis láctica ocasionados por la metformina se presentan por no haberse prestado atención a las contraindicaciones.

La metformina se empezó a vender en los Estados Unidos de América en 1995. Durante el primer año se notificaron a la Administración de Alimentos y Medicamentos (FDA) 47 casos de acidosis láctica atribuibles al uso del fármaco.

Las siguientes son las contraindicaciones mencionadas en el resumen de las características del producto:
- Trastorno renal (aunque sea moderado).

- Insuficiencia respiratoria o cardíaca; episodio reciente de infarto agudo del miocardio.

- Lesión hepatocelular; intoxicación por alcohol.

- Riesgo de alteración de la función renal, como en casos de deshidratación, infecciones graves, acidosis diabética o cetoacidosis (en estos casos es necesario suspender el medicamento).

- Inyección de medios de contraste yodados (la metformina debe suspenderse 48 horas antes de la inyección y dejar de administrarse durante 48 horas después de ella debido al riesgo de insuficiencia renal aguda).

Suele recomendarse que se interrumpa el consumo de metformina 48 horas antes de cualquier procedimiento quirúrgico electivo.

\section{Riesgo de infección, sepsis y muerte asociado con el uso de etanercepto $\left(\right.$ Enbrel $^{\circledR}$ ) (Estados Unidos de América)}

La Administración de Alimentos y Medicamentos (FDA) ha dado a conocer a los médicos algunos datos nuevos de carácter alarmante sobre el uso de etanercepto (Enbrel ${ }^{\circledR}$ : Immunex y Wyeth-Ayerst). Los informes generados después de la comercialización del producto indican que algunos pacientes tratados con él han sufrido infecciones graves, en algunos casos hasta sepsis, y que varios han fallecido como resultado de estas infecciones.

El etanercepto es una proteína obtenida por manipulación genética que se autorizó el pasado noviembre para el tratamiento de pacientes con síntomas moderados a graves de artritis reumatoide activa que no han respondido a otros tratamientos. El fármaco puede aliviar notablemente el dolor y la inflamación articulares que sufren estos pacientes. El rótulo indica que no debe administrarse a pacientes con sepsis y que debe suspenderse en pacientes que contraen una infección grave. A la luz de los datos más recientes, la ad- 
vertencia en conexión con la sepsis se ha ampliado de tal modo que ahora se incluye en ella a pacientes con cualquier tipo de infección activa, aun tratándose de una infección crónica o localizada. Actualmente también se recomienda que los pacientes que contraen una infección mientras están en tratamiento con etanercepto sean observados muy cuidadosamente. Se recomienda que los médicos sean precavidos al contemplar la prescripción de etanercepto a pacientes con antecedentes de infecciones recurrentes o con trastornos subyacentes que podrían aumentar su susceptibilidad a sufrir una infección, tales como diabetes avanzada o mal controlada.

A pesar de que muchos pacientes con artritis reumatoide son propensos a las infecciones, se sigue temiendo que el etanercepto los predisponga aún más a las infecciones graves. Muchas de las infecciones se presentaron poco tiempo después de iniciarse la administración de este medicamento. El etanercepto inhibe la actividad del factor de necrosis tumoral, sustancia que forma parte de las defensas naturales del organismo contra infecciones graves.

Hasta la fecha, los ensayos clínicos controlados no han mostrado un aumento de las infecciones graves en pacientes que reciben etanercepto. La FDA ha pedido al fabricante que lleve a cabo estudios adicionales con el fin de evaluar el riesgo de infección grave en conexión con el tratamiento con este fármaco. Mientras tanto, por precaución los médicos deben tener presentes los últimos informes de reacciones adversas a la hora de evaluar los riesgos y beneficios del etanercepto.

\section{POLÍTICA SOBRE MEDICAMENTOS}

\section{Se establecen los reglamentos finales con respecto al uso de protectores contra los rayos solares (Estados Unidos de América)}

La Administración de Alimentos y Medicamentos (FDA) ha terminado de definir sus reglamentos en torno al uso de protectores solares de venta libre. Estos reglamentos (que llevan el nombre de monografía final) enumeran los ingredientes activos que pueden usarse en estos productos y establecen los requisitos para la rotulación y los ensayos clínicos. Los reglamentos piden que la rotulación de todos los productos de venta libre que confieren protección contra los rayos solares sea uniforme y fácil de interpretar, con el fin de orientar a los consumidores en sus decisiones sobre el uso de estos productos.

Debido a los requisitos para efectuar ensayos clínicos y a la naturaleza estacional del uso de estos productos, los fabricantes tendrán 24 meses para cumplir con los nuevos reglamentos. Los fabricantes de cosméticos bronceadores que no contienen protectores solares tendrán 12 meses para añadir la advertencia requerida a sus productos. No obstante, se alienta a todos los fabricantes a adoptar la nueva rotulación lo antes posible.

Las recomendaciones con respecto al uso de protectores contra rayos ultravioleta tipo A (UVA) serán las mismas que existen actualmente hasta que la agencia examine el asunto con más detenimiento.

\section{La FDA lucha contra la venta por Internet de medicamentos adquiribles exclusivamente con receta (Estados Unidos de América)}

La Administración de Alimentos y Medicamentos (FDA) ha ideado una estrategia para combatir la venta ilegal por Internet de medicamentos que solo se pueden adquirir con receta. Uno de los principales componentes de dicha estrategia consiste en la creación de nuevas alianzas de cooperación con las agencias estatales y nacionales encargadas de imponer el cumplimiento de la ley y con las entidades reguladoras a fin de poder combatir más eficazmente el problema de las ventas ilegales por Internet.

De acuerdo con la ley federal y las leyes estatales, todo paciente debe ser sometido a examen físico por un trabajador de la salud autorizado antes de recibir una receta por primera vez. El paciente, con la receta debidamente llenada, se dirige a un farmacéutico registrado en una farmacia autorizada que cumpla los requisitos estatales de funcionamiento. Si bien es cierto que las farmacias acreditadas que funcionan en línea pueden ser de gran utilidad para los consumidores - muchas permiten incluso que los pacientes consulten a farmacéuticos desde su propia casa-, se pueden crear sitios farmacéuticos en la Web cuyo aspecto inspira confianza cuando en la realidad tanto el vendedor como los productos son fraudulentos. La estrategia de la FDA ha sido impulsada por el enorme número de sitios en la Web - tanto en los Estados Unidos de América como en otros países- que venden medicamentos potentes sin recetas válidas o sin una interacción productiva con un médico u otro profesional de la salud. A menudo las ventas se basan solamente en la contestación de un cuestionario por parte del consumidor. Los pacientes que entran en estas compras ilícitas corren el riesgo de sufrir efectos secundarios ocasionados por el uso de medicamentos prescritos indebidamente y de sufrir daños por el consumo de fármacos contaminados, falsificados o vencidos.

\section{Nuevos anuncios de la FDA que declaran ilegal la venta de productos de tabaco a menores de edad}

El 2 de agosto de 1999 la Administración de Alimentos y Medicamentos (FDA) lanzó una nueva campaña publicitaria en cinco estados y 11 ciudades adicionales para lograr mayor adhesión al reglamento de la FDA que declara ilegal la venta de productos de tabaco a menores de edad.

Por primera vez, la FDA transmitirá anuncios durante el año entero en todo el territorio de los estados de Colorado, Tennessee, Nueva Hampshire, Michigan y Nevada. Estos anuncios también se transmitirán en 11 ciudades adicionales: Bridgeport, Connecticut; Lafa- 
yette, Luisiana; San Antonio, Texas; Springfield, Misuri; Rockford, Illinois; Rochester, Nueva York; Roanoke, Virginia; Lawton, Oklahoma; Augusta, Maine; Washington, DC y Macon, Georgia.

La campaña publicitaria, que tiene un costo de US\$5000000, llegará a 24 millones de jóvenes y se encamina a alentar a los propietarios de negocios y vendedores a poner de su parte para evitar que la juventud compre productos a base de tabaco (cigarrillos y tabacos de mascar). Esta campaña sencilla pero poderosa, que se denomina "For a Reason" [Hay motivos], se transmite por la radio, por los medios de comunicación impresos, y al aire libre. También se está poniendo a prueba un anuncio de televisión de 30 segundos en tres lugares diferentes.

Los anuncios forman parte de un programa educacional general de la FDA llamado "No ID, No Smokes" [Sin identificación no hay tabaco] que se inició en 1997 con objeto de proporcionar a los vendedores los medios y conocimientos necesarios para cumplir con los reglamentos de la FDA sobre la venta de productos del tabaco. A través de este programa, la FDA proporciona gratuitamente estuches informativos y rótulos que se colocan dentro de los establecimientos para recordarles a los gerentes y empleados que pidan ver alguna forma de identificación y que les expliquen a los clientes por qué se les está pidiendo que se identifiquen.

\section{Registro y control de productos medicinales naturales (Ecuador)}

En mayo de 1999, el Gobierno de Ecuador publicó en su Registro Oficial 186 el Acuerdo Ministerial 1281 que expide las normas y procedimientos para el registro y control de productos naturales de uso medicinal y de los establecimientos donde se fabrican, almacenan y comercializan estos productos. El Acuerdo fue firmado por el Ministro de Salud Pública el 16 de abril.

\section{USO RACIONAL}

\section{Uso inapropiado de suplementos \\ dietéticos orales en forma líquida (Estados Unidos de América)}

Los suplementos dietéticos orales en forma líquida a menudo se administraban de forma inespecífica a residentes de hogares para ancianos que comían poco y perdían peso, según un estudio publicado en el número de 1998 de la Journal of the American Geriatrics Society. De 29 personas a quienes se prescribieron estos suplementos, solamente nueve recibieron el tipo adecuado en la cantidad debida. En general, $55,1 \%$ de los suplementos prescritos se consumieron, pero casi la mitad de los pacientes siguieron perdiendo peso. Según los autores, "Se prescribieron los suplementos sin haber investigado los factores subyacentes que contribuían a la pérdida de peso ..."

\section{Coumadin ${ }^{\circledR}$ y Marevan ${ }^{\circledR}$ no son intercambiables (Australia)}

La warfarina se vende con dos marcas: Coumadin ${ }^{\circledR}$ y Marevan ${ }^{\circledR}$. Couma$\operatorname{din}^{\circledR}$ viene en comprimidos de 1,2 y 5 mg y Marevan ${ }^{\circledR}$, en comprimidos de 1, 3 y 5 mg. Recientemente el Comité Asesor en Reacciones Medicamentosas Adversas (ADRAC) recibió un informe sobre una respuesta terapéutica intensificada en asociación con el uso de warfarina de marca Coumadin ${ }^{\circledR}$. El problema surgió cuando un paciente que había estado en tratamiento con Marevan ${ }^{\circledR} 2 \times 1 \mathrm{mg}$ recibió $2 \mathrm{mg}$ de warfarina. Solamente Coumadin ${ }^{\circledR}$ viene en esta dosis y, después de administrado el medicamento, la razón normalizada internacional (International Normalized Ratio, INR) del paciente subió de su valor estable habitual de 2 a más de 5 . El informe indicó que se trataba del tercer caso de este tipo en los 18 meses anteriores. El ADRAC insiste en la importancia de dar a conocer a los prescriptores que no está demostrada la equivalencia biológica de Cou- madin ${ }^{\circledR}$ y Marevan ${ }^{\circledR}$ y que por lo tanto estos fármacos no son intercambiables.

\section{Debe descartarse el embarazo antes de prescribir clomifeno (Estados Unidos de América)}

El clomifeno se ha usado por varios decenios principalmente para inducir la ovulación. Los niños que nacen como resultado de la inducción de la ovulación por clomifeno no parecen sufrir ningún tipo de trastorno. No obstante, los resúmenes de las características de los productos (SPC) a base de clomifeno describen claramente un efecto teratógeno en animales, en particular defectos oculares en ratas y anomalías esqueléticas y cerebrales en conejos.

Según los SPC, se sospecha un efecto teratógeno en mujeres, lo cual significa que se proscribe la administración de clomifeno después de la concepción. Aunque el clomifeno se ha usado por decenios, hay muy pocos datos sobre el riesgo de efectos teratógenos en etapa temprana del embarazo. No puede descartarse un aumento moderado del riesgo de malformaciones, especialmente de tipo esquelético.

Lo anterior subraya la necesidad de descartar el embarazo en toda candidata al tratamiento con clomifeno. Los embarazos que inadvertidamente se exponen al clomifeno deben ser vigilados con periodicidad, sobre todo con ecografía.

\section{RETIROS DEL MERCADO}

\section{Retiro voluntario del sulfato de gentamicina inyectable (en viales de USP $40 \mathrm{mg} / \mathrm{mL}$ y de $80 \mathrm{mg} / 2 \mathrm{~mL}$ )}

ESI Lederle ha retirado voluntariamente del mercado 20 lotes de sulfato de gentamicina inyectable en concentraciones de $40 \mathrm{mg} / \mathrm{mL}$ y de $80 \mathrm{mg} /$ $2 \mathrm{~mL}$ debido a un número inesperadamente alto de manifestaciones adversas (reacciones del tipo suscitado por 
pirógenos, con fiebre, escalofríos o disnea). Estas manifestaciones se han notificado en pacientes que han recibido medicamentos de estos lotes, a pesar de que cumplen todas las especificaciones establecidas para el producto.

\section{Retiro del astemizole (Hismanal ${ }^{\circledR}$ ) (Estados Unidos de América)}

Janssen Pharmaceutica advierte a los proveedores de atención de salud que suspenderá voluntariamente la fabricación, distribución y venta de los comprimidos de Hismanal ${ }^{\circledR}$ de $10 \mathrm{mg}$. La compañía ha tomado esta determinación después de estudiar detenidamente la familia de los antihistamínicos, que abarca muchas otras opciones terapéuticas.

\section{El colutorio DawnMist ${ }^{\circledR}$ (Estados Unidos de América)}

La compañía Donovan Industries, Inc. está retirando todos los lotes del colutorio DawnMist ${ }^{\circledR}$ fabricados en Malasia por sospecharse que el producto pueda estar contaminado con Burkholderia cepacia. El enjuague viene en envases de plástico blancos de 2 y 4 oz. y se distribuyó por todo el país en hospitales y otros centros médicos. Burkholderia cepacia es un patógeno nosocomial que puede ocasionar neumonía asociada con el uso de aparatos de ventilación artificial.

\section{La poligelina (Haemaccel ${ }^{\circledR}$ ) y su asociación con hipotensión arterial (Alemania)}

El Instituto Federal de Medicamentos y Dispositivos Médicos ha emitido una advertencia urgente sobre el retiro por el fabricante (Hoechst Marion Roussel) de todos los lotes de poligelina (Haemaccel $\left.{ }^{\circledR} 35\right)$, producto usado para aumentar el volumen plasmático, después de recibir un número mayor del esperado de informes de casos de hipotensión en conexión con su administración. Algunos de estos informes se describieron como poco característicos, por tratarse de una caída brusca de la tensión arterial sin signos de alergia. No obstante, no se ha establecido una conexión explícita con la poligelina. La evaluación y vigilancia de estos casos están en marcha todavía, pero por precaución el fabricante ha iniciado la retirada del producto de todos los países donde se distribuye.

\section{Retiro de productos del mercado en 1999 (Argentina)}

Durante el mes de junio de 1999, la Asociación Nacional de Medicamentos, Alimentos y Tecnología Médica sancionó las disposiciones que ordenaban el retiro del mercado de diversos productos no aptos para el uso o consumo. Entre estas figuran las siguientes:

Disposición no. 2894/99 (1 de junio). Medicamento no legítimo: Mentholatum ungüento, en cuyo rótulo aparecen los siguientes datos: "Fabricado y garantizado por The Mentholatum Co., Inc., Buffalo, Nueva York, Estados Unidos de América; fraccionado en Paraguay; representante exclusivo Luis Cassanello, S.A.I.C.E.C.A." Realizadas las verificaciones correspondientes, se observó que la especialidad medicinal en cuestión carece de número de lote, fecha de vencimiento y certificado que autorice su comercialización.

Disposición no. 3125/99 (15 de junio). Medicamento no legítimo: Producto rotulado como Bayaspirina $\times 100$ comprimidos (lote 704031 con vencimiento en 09/01). El titular del certificado de la especialidad medicinal en cuestión es la firma Bayer, S.A. Realizados los controles correspondientes, se comprobó que la partida en cuestión no es legítima, por lo que se prohibió su comercialización y uso en todo el país.

Disposición no. 3127/99 (15 de junio). Medicamento no legítimo: Preparación denominada wira sacha, extracto de plantas medicinales que, en la etiqueta interior del envase, dice ser producto yeerva vidaa-producto peruano. Realizadas las verificaciones correspondien- tes, se comprobó que la preparación mencionada no cuenta con certificado de autorización por parte de esta Administración nacional. En consecuencia, se prohibió su comercialización y uso en todo el país.

Disposición no. 3220/99 (28 de junio). Medicamento no legítimo: Producto rotulado como Novalgina ${ }^{\circledR}$, gotas $\times$ $10 \mathrm{~mL}$ (lote 0211 con vencimiento en 04/00), marca registrada de la firma Laboratorios Hoechst del Paraguay, S.A. Realizadas las verificaciones correspondientes, se comprobó que la partida en cuestión era un producto original elaborado en la planta de la empresa mencionada y que ingresó al país de contrabando. En consecuencia, se prohibió su comercialización y uso en todo el territorio nacional.

Disposición no. 3275/99 (28 de junio). Medicamento no legítimo: Producto sertralina $\left(\right.$ Zoloft $\left.^{\circledR}\right)$, cápsulas de $50 \mathrm{mg}$. (lote 80772048 con vencimiento en 03/200), de los laboratorios Pfizer de la industria italiana. Realizadas las verificaciones correspondientes en relación con dicha partida, se comprobó que, en un extremo del blíster de los envases primarios, figuraba el vencimiento con fecha 03/2000. Por otra parte, en el frente del blíster y marcada con impresión indeleble se encontraba la leyenda "vence en marzo del 2000". Asimismo, en el envase secundario, debajo del número de lote, una serie de cruces tapaban parcialmente la fecha 09/2003 y aparecía, también marcada con impresión indeleble, la fecha 03/2000. Días después, en una entrevista con el director técnico de la firma Pfizer S.R.L., este alegó que la partida provenía de Italia y que su plazo de vencimiento era mayor. Agregó que, por ese motivo, procedieron a remarcar la fecha que fue otorgada en el momento del ingreso. El Instituto Nacional de Medicamentos, por su parte, aclaró que la Administración Nacional autorizó la extensión del vencimiento a 60 meses, con fecha posterior a la importación. Por todo lo expuesto, y teniendo en cuenta que la anomalía detectada podría dar lugar a confusión por parte de los consumidores y a un consecuente riesgo para su salud, se prohibió la co- 
mercialización y uso del lote en cuestión en todo el país.

\section{Falsificación de Distensil ${ }^{\circledR}$ y Buscapina Compositum ${ }^{\circledR}$ N (Perú)}

En el Perú se ha detectado la falsificación de la especialidad farmacéutica Distensil $^{\circledR} 500 \mathrm{mg}$ inyectable, fabricada por Laboratorios Trifarma, S.A. El principio activo original es el cloruro de suxametonio, relajante muscular utilizado como coadyuvante de la anestesia y administrado solamente por médicos anestesiólogos en centros quirúrgicos.

El análisis del producto decomisado ha permitido determinar que los viales decomisados son falsificados y no contienen cloruro de suxametonio. Entre las características que permiten su diferenciación del original resaltan la mala rotulación y la impresión poco legible, así como una menor altura en el nivel del contenido.

En el Perú se ha detectado la falsificación del producto Buscapina Compositum ${ }^{\circledR} \mathrm{N}$ en su presentación de caja con 20 comprimidos recubiertos, fabricado por Boehringer Ingelheim, S.A. La composición autorizada para este producto abarca los principios activos paracetamol y N-butilbromuro de hioscina.

El examen del producto decomisado ha permitido determinar que los comprimidos analizados no contienen esos principios activos. Este producto, a diferencia del original, lleva el nombre de Buscapina Compositum ${ }^{\circledR}$ NF y viene en una caja de distinto tamaño con un número diferente de registro sanitario y sin ninguna hoja descriptiva dentro del envase ni el nombre del distribuidor.

\section{ENMIENDAS A LA ROTULACIÓN}

\section{Nueva rotulación y cambios en las indicaciones sobre el uso de troglitazona (Rezulin ${ }^{\circledR}$ ) (Estados Unidos de América)}

Se están haciendo modificaciones importantes en la rotulación y en los usos recomendados de la troglitazona
(Rezulin ${ }^{\circledR}$ ), fármaco que se utiliza para el tratamiento de la diabetes mellitus tipo II (la no insulinodependiente o forma adulta). Estas modificaciones se han hecho porque nuevos datos (es decir, pruebas adicionales de daño hepático grave y en ocasiones mortal en pacientes tratados con Rezulin ${ }^{\circledR}$ ) indican que el medicamento debe administrarse solo a pacientes cuya diabetes no puede controlarse satisfactoriamente con otros tratamientos y nunca como agente único inicial para el tratamiento de la diabetes tipo II. Los cambios en la rotulación también abarcan algunas recomendaciones sobre la necesidad de vigilar más de cerca la función hepática en pacientes que reciben Rezulin ${ }^{\circledR}$ :

- Rezulin ${ }^{\circledR}$ ya no está indicado como agente terapéutico único de entrada.

- Los pacientes a quienes se piensa administrar Rezulin ${ }^{\circledR}$ deben ser sometidos a pruebas de función hepática antes de iniciarse el tratamiento, y posteriormente cada mes durante el primer año del mismo (y no solo un total de 10 veces como se recomendaba antes). Después del primer año de tratamiento se deben realizar las pruebas cada trimestre (y no solo periódicamente, como solía recomendarse).

Además de los cambios de rotulación aquí descritos, se añadirá una nueva indicación al rótulo sobre el uso de Rezulin ${ }^{\circledR}$ en combinación con las sulfonilureas y la metformina en pacientes cuya diabetes no puede controlarse satisfactoriamente mediante el uso combinado de estos otros agentes hipoglucemiantes. Esta indicación está basada en datos clínicos recientes.

\section{DECISIONES DIVERSAS}

\section{La FDA aprueba nuevo medicamento para el cáncer cerebral (Estados Unidos de América)}

La FDA ha aprobado el uso de temozolomida, tratamiento antineoplásico, en pacientes adultos con astrocitoma anaplásico (tumor cerebral) que han sufrido una recaída tras la administración de quimioterapia a base de derivados de la nitrosourea (carmustina o lomustina) y procarbazina.

En los Estados Unidos de América se diagnostican cada año alrededor de 18000 casos nuevos de cáncer intracraneano primario (es decir, cáncer cerebral), cifra que representa cerca de $2 \%$ de todos los cánceres en adultos. Más de $50 \%$ de estos tumores son gliomas de alto grado (i.e., glioblastomas multiformes y astrocitomas anaplásicos). Los pacientes con estos tumores a menudo tienen discapacidades muy graves, tales como falta de coordinación motora, convulsiones de origen epiléptico y anomalías visuales. La aprobación de la temozolomida ofrece a los pacientes una opción terapéutica adicional cuando no se observa respuesta al tratamiento inicial con radioterapia y quimioterapia.

La temozolomida ha recibido autorización acelerada gracias a un mecanismo regulador que permite la autorización temprana de productos para el tratamiento de trastornos muy graves o mortales cuando no se conoce ningún tratamiento eficaz o cuando el nuevo producto aventaja notablemente en eficacia a los otros tratamientos disponibles. La autorización acelerada se basa en marcadores de eficacia "sustitutivos", tales como la reducción de la masa tumoral, y no en un efecto clínico documentado sobre la supervivencia o calidad de la vida.

\section{La FDA aprueba el uso de una proteína de fusión para el tratamiento de linfomas (Estados Unidos de América)}

La Administración de Alimentos y Medicamentos (FDA) ha autorizado el uso de una proteína de fusión para el tratamiento de una forma de cáncer poco frecuente que no responde a otros regímenes terapéuticos. Se trata de la diftitoxina denileuquina $\left(\right.$ Ontak ${ }^{\circledR}$, Seragen, Inc.), que se autorizó en febrero para uso en pacientes con linfoma cutáneo de células $\mathrm{T}$ crónico o recurrente.

Entre los efectos secundarios asociados con el uso de diftitoxina denileu- 
quina figuran la fiebre y síntomas de gripe en $91 \%$ de los pacientes; reacciones de hipersensibilidad agudas en 69\%; náusea y vómitos en $64 \%$; infecciones en $48 \%$ y síndrome de filtrado vascular en $27 \%$.

La diftitoxina denileuquina viene en solución congelada estéril. Cada vial para una sola administración contiene $2 \mathrm{~mL}$ de diftitoxina denileuquina a una concentración de $150 \mu \mathrm{g} / \mathrm{mL}$.

\section{La talidomida (Thalomid ${ }^{\circledR}$ ): medicamento huérfano contra la enfermedad de Crohn (Estados Unidos de América)}

La talidomida $\left(\right.$ Thalomid $^{\circledR}$ ) ha sido designada por la Administración de Alimentos y Medicamentos (FDA) como medicamento huérfano para el tratamiento de la enfermedad de Crohn. El fármaco, elaborado por Celgene Corp., está actualmente a prueba en ensayos clínicos de fase II en pacientes con enfermedad de Crohn y su uso ya ha sido autorizado en pacientes leprosos.

"Una producción excesiva de factor tumoral necrotizante puede ocasionar inflamación intensa en casos de enfermedad de Crohn, artritis reumatoide y otras enfermedades autoinmunitarias", afirma un representante de Celgene Corp. "La talidomida tiene efectos inmunomoduladores que podrían asociarse con la supresión de la producción excesiva de factor de necrosis tumoral tipo alfa".

\section{La FDA autoriza la rosiglitazona para el tratamiento de la diabetes mellitus tipo II \\ (Estados Unidos de América)}

La FDA ha aprobado la rosiglitazona (Avandia ${ }^{\circledR}$ ), medicamento recién elaborado que pertenece al grupo de las tiazolidinedionas para el tratamiento de la diabetes mellitus tipo II (también conocida por diabetes no insulinodependiente o del adulto). Este fármaco ha sido aprobado para pacientes con este tipo de diabetes que no reciben insulina. Los pacientes que estén en tratamiento con rosiglitazona también deben mantenerse en un peso adecuado y seguir una dieta rigurosa.

El nuevo fármaco mejora un trastorno que parece ser causa subyacente de diabetes del adulto: la resistencia del organismo a la insulina. En ensayos clínicos con más de 4000 pacientes previamente controlados a base de dieta solamente o con metformina, la rosiglitazona mejoró la capacidad de utilizar la insulina endógena.

En general, la rosiglitazona fue bien tolerada en los ensayos clínicos. Las reacciones adversas más notificadas fueron la infección, el dolor y la cefalea, pero estos síntomas ocurrieron con una frecuencia similar a la observada en pacientes que tomaron placebo. También hubo casos de edema leve o moderado, aumento del colesterol plasmático y anemia en pacientes tratados con rosiglitazona, pero no fueron lo suficientemente graves para suspender el tratamiento.

Otro medicamento de la familia de las tiazolidinedionas, conocido por Rezulin $^{\circledR}$ (troglitazona, fabricada por Parke-Davis), se ha asociado con hepatotoxicidad idiosincrática o insuficiencia hepática. Esta puede ocurrir en cualquier momento durante el tratamiento con este fármaco y ha llegado a ocasionar la muerte en algunos casos y en otros la necesidad de efectuar un transplante de hígado. En cambio, en los ensayos clínicos efectuados con pacientes tratados con rosiglitazona no ha habido ningún indicio de hepatotoxicidad de origen medicamentoso.

Pese a lo antedicho, debido a la hepatotoxicidad asociada con Rezulin ${ }^{\circledR}$ la FDA recomienda que se observen las enzimas hepáticas en pacientes en tratamiento con rosiglitazona. Dichas enzimas deben medirse cuando se inicia el tratamiento y posteriormente cada trimestre durante el primer año. En años subsiguientes las pruebas deben efectuarse periódicamente.

\section{El uso del tiomersal en vacunas (Estados Unidos de América)}

El tiomersal se ha usado como sustancia aditiva en los productos biológi- cos y en las vacunas desde los años treinta debido a su gran efecto bactericida contra las bacterias usadas en muchas vacunas y a su capacidad para prevenir la contaminación bacteriana, especialmente en recipientes abiertos que contienen varias dosis. Muchas de las vacunas que se recomiendan para niños estadounidenses, aunque no todas, contienen tiomersal. No obstante, ante la posibilidad de que el tiomersal conlleve algún riesgo, el Servicio de Salud Pública (PHS) de los Estados Unidos de América, la Academia Estadounidense de Pediatría (AAP) y los fabricantes de vacunas coinciden en que las vacunas que contienen tiomersal deben suspenderse tan pronto sea posible. Se llegó a conclusiones similares este año en una reunión a la que asistieron representantes de las agencias reguladoras y de los fabricantes de vacunas en Europa y de la Administración de Alimentos y Medicamentos (FDA). En la reunión se examinó el uso de vacunas con tiomersal elaboradas o vendidas en países europeos.

El PHS y la AAP están tratando de lograr, mediante un esfuerzo conjunto, que las vacunas que contienen tiomersal sean reemplazadas a la mayor brevedad, procurando conseguir al mismo tiempo que se mantengan en toda la población infantil nuestros altos niveles de cobertura por vacunación y la poca morbilidad que con ellos se asocia.

\section{PUBLICACIONES}

WHO Expert Committee on Specifications for Pharmaceutical Preparations: Thirty-fifth report. Ginebra: Organización Mundial de la Salud; 1999. (Serie de informes técnicos 885). Precio: FS 35,00; FS 24,50 en países en desarrollo. ISBN 9241208856

Actualmente se están preparando las versiones de esta obra en francés y español. La versión en inglés puede pedirse directamente a: WHO, Distribution and Sales, 1211 Geneva 27, Suiza. Tel.: (4122) 7912476; fax: (4122) 7914857. Correo electrónico: Publications@who.ch 


\section{REFERENCIAS}

American Pharmaceutical Association. Pharmacy Today. Vol. 5, No. 1, enero de 1999.

Asociación Nacional de Medicamentos, Alimentos y Tecnología Médica. Informe (Argentina). 10 de julio de 1999.

Australian Adverse Drug Reactions Bulletin. Vol. 18, No. 2, junio de 1999.

Butlletí Groc (Barcelona). Vol. 12, No. 2, marzoabril de 1999.

Dirección General de Medicamentos, Insumos y Drogas. Alerta (Perú). Marzo y abril de 1999.

Food and Drug Administration. Talk Paper T 9923, 21 de mayo de 1999.

Food and Drug Administration. Talk Paper T 9925, 26 de mayo de 1999.

Food and Drug Administration. Talk Paper T 9926, 9 de junio de 1999.

Food and Drug Administration. Talk Paper T 9928, 16 de junio de 1999.

Food and Drug Administration. Talk Paper P 9915, 30 de julio de 1999.

Food and Drug Administration. Talk Paper T 9935, 02 de agosto de 1999.
Food and Drug Administration. Talk Paper T 9937, 11 de agosto de 1999

Journal of the American Pharmaceutical Association. Vol. 39, No. 4 (julio/agosto)

Market News Service. Pharmaceutical Raw Materials/Essential Drug Report (Geneva). No. 87 (junio de 1999).

Préscrire International. Vol. 8, No. 40, abril de 1999.

Préscrire International. Vol 8., No. 41, junio de 1999.

RED Directions Vol. 5, No. 5, mayo de 1999.

Registro Oficial No. 186 (Ecuador) 7 de mayo de 1999.

WHO DRS Information Exchange System. Alert. No. 81, 3 de marzo de 1999.

WHO DRS Information Exchange System. Alert. No. 83, 13 de mayo de 1999.

WHO DRS Information Exchange System. Alert. No. 85, 28 de mayo de 1999.

WHO DRS Information Exchange System. Alert. No. 87, 19 de julio de 1999.

World Health Organization. WHO Expert Committee on Specifications for Pharmaceutical Preparations: Thirty-fifth Report. Ginebra: WHO; 1999. (Technical Report Series No. 885).
Información farmacológica da a conocer las decisiones oficiales sobre regulación de productos farmacéuticos adoptadas por organismos gubernamentales e internacionales en todo el mundo, los fundamentos científicos en que se sustentan tales decisiones y otros datos de interés relacionados con el tema. Como la mayor parte de la información proviene de fuentes de circulación relativamente limitada, su diseminación en esta forma permite hacerla llegar a un público más amplio. De esta manera se pretende contribuir a limitar el uso irracional de medicamentos y fomentar su uso racional en la Región de las Américas. La sección está a cargo del Programa de Medicamentos Esenciales y Tecnología para la Salud (HSE) de la OPS y se publica en la Revista Panamericana de Salud Pública Pan American Journal of Public Health en enero, abril, julio y octubre. Las separatas pueden solicitarse al programa mencionado, Organización Panamericana de la Salud, 525 Twenty-third Street, NW, Washington, DC 20037, EUA. 